\title{
Differential Attainment in Career Progression of Doctors in the UK
}

\begin{abstract}
:
Differential attainment in career progression in the NHS is a complex issue with many interplaying factors apart from individual protected characteristics. In this paper, we examine the attainment gap, causes for these disparities and some recommendations to reduce the gap.
\end{abstract}

Our review shows that there is significant DA between groups of doctors on the basis of gender, ethnicity, race and country of primary medical qualification. The likely causes are bias, lack of opportunity, poor supervision, mentorship, sponsorship, dichotomous treatment of doctors based on training or non-training status and cultural exclusion. Data is not monitored or reported and there is little organisational accountability. Solutions are likely to include transparent data on recruitment as well as progression for benchmarking, training support for all doctors, initiatives which are sensitive to gender, parental responsibility, cultural heritage, language and robust supervision including mentorship and sponsorship.

This scoping review forms part of the Alliance for Equality in Healthcare Professions project on Differential Attainment chaired by the British Association of Physicians of Indian origin (BAPIO) and will be integrated into the Bridging the Gap project undertaken by BAPIO Institute for Health Research (BIHR). This work is part of six domains of doctors' careers in the NHS.

Keywords; Differential attainment, Career progression, NHS

\author{
Veena Daga ${ }^{1}$, Robert Blizzard ${ }^{2}$, Anshoo \\ Dhelaria $^{3}$, Saraswati Hosdurga ${ }^{4}$, Saba \\ Hussain ${ }^{4}$, Spandana Madabhushi ${ }^{3}$, \\ Geraldine Sawney ${ }^{3}$, Arvind Shah ${ }^{5}$, \\ Lambrini Theocharidou ${ }^{6} \&$ Rupal \\ Shah ${ }^{10}$ \\ ${ }^{1}$ Leeds Teaching Hospitals NHS Trust, ${ }^{2}$ \\ Health Education England Yorkshire and \\ Humber, ${ }^{3}$ Lister Hospital, ${ }^{4}$ CCHP, Sirona \\ Care and Health, Bristol, Bristol Children \\ Hospital, ${ }^{5}$ North Middlesex University \\ Hospital, London, 6 Countess of Chester \\ Hospital, ${ }^{9}$ HEE London and GP Battersea, \\ London \\ dme.bta@bapio.co.uk
}

Cite as: Daga, V., Blizzard, R., Dhelaria, A., et. al. (2021) Differential attainment in career progression of doctors in the UK. Sushruta J Health Policy \& Opinion vol 14; Issue 1: 1-16 ePub 28.12.2020 DOI: https://doi.org/10.38192/14.1.8

\section{Article Information}

Submitted 17.12.2020

Published 28.12.2020

Open Access- Creative Commons Licence International $\mathrm{v} 4.0$

\section{Introduction}

A career in medicine is one of the most sought-after options for school leavers, even though the trajectory towards becoming a General Practitioner (GP) or a Hospital consultant is long and arduous. It involves multiple postgraduate exams and many additional years of training after leaving medical school. Despite this, the medical profession typically attracts motivated, hardworking and enthusiastic individuals, who make considerable sacrifices not only to gain a place at medical school, during postgraduate training and through a firm commitment to the cycle of life-long learning, appraisals and recertification/ renewal of the licence to practice. It is well known that the working environment can be demanding and stressful, often involving the need to work for long, unsociable hours, compared to many other professions. Due to the length and complexity of medical training pathways, there are many barriers and facilitators with regard to career progression. 


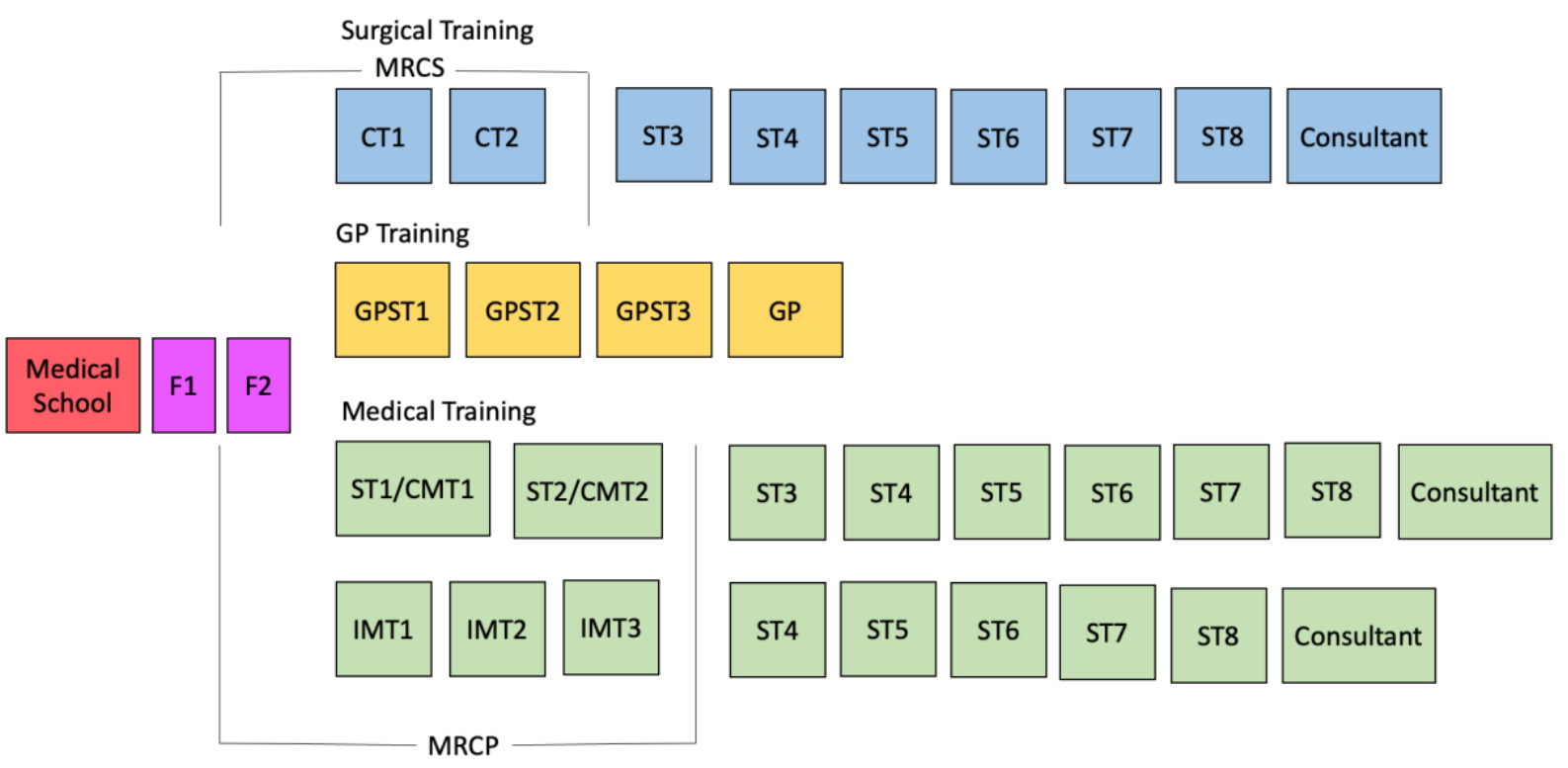

Figure 1. A simplified chart of doctors' career pathways in the UK (1)

The illustration in figure 1, shows the common career pathways for postgraduate training in the UK, but it is important to note that there are several other bespoke pathways (2)(3)(Sheikh, 2017). Additionally, doctors can opt to pause their training programme in discussion with their supervisors through an Out-of-Programme (OOP) experience. Doctors often choose an OOP due to personal reasons, specialist clinical placements, fellowships and in research, leadership or additional qualifications. (4)(5) Doctors in the Specialist and Associate Specialist (SAS) grades and Locally Employed Doctors (LED) aim to follow similar and equivalent pathways as per their specialty, but are normally excluded from the infrastructure that supports those in formal 'training'. (6)

It has become apparent over recent decades, that particular demographic factors influence career progression and create an attainment gap between groups based on certain (protected) characteristics. This rapid scoping review was conducted as part of the 'Bridging the Gap' project (7) by the British Association of Physicians of Indian Origin (BAPIO). The objective is to summarise key literature around differential attainment (DA) in career progression of doctors working in the UK National Health Service (NHS). This review aims to identify and propose solutions to redress the gap. In this paper, we address four broad aspects: the scale, impact, potential causes and suggest some solutions.

\section{Methods:}

In this review, we used search engines - Google Scholar and PubMed using the key terms: 'career progression'; 'differential attainment'; 'medical professional'; doctors; and 'the NHS'. Reports from organisations (such as GMC, Royal colleges and Health Education England -HEE) were also reviewed. The protected characteristics we deemed most relevant were: gender, ethnicity, country of primary medical qualification, disability and socioeconomic background. Additionally, it appeared that the type of career pathway (training versus non-training, full time versus less-than-full time LTFT) influenced career progression. All relevant publications and reports identified, were subsequently divided among the ten authors who reviewed the papers and screened them based on agreed criteria.

The definition of DA in career progression was agreed between the authors. DA is the "observed gap in the achievements of different cohorts of individuals based on factors beyond their individual ability" (7). Career progression was defined as one's evolution within one's chosen specialty. Therefore, we excluded papers and reports focusing on undergraduate medical students. Although career progression is affected by examination success and recruitment aspects, these areas were looked at in detail by other groups in the 'Bridging the Gap' project. Therefore papers whose main focus was on assessment and recruitment were also excluded.(7)

During the initial screening of the evidence, the authors also decided under which category (scale and impact, causes and solutions) each paper or report was categorised. Some of them could be placed in more than one category. Then, groups were formed with at least one consultant or educator and one trainee to look at each of the three categories. 


\section{Results}

\section{Scale and Impact}

Our review demonstrated a significant differential between doctors in the rate of career progression, access to training posts, geographical variation (rural vs metropolitan), variation by specialty and more often than not, these appeared to be determined by certain non-academic characteristics of groups of doctors. Demographic factors such as gender, race or ethnicity and country of primary medical qualification (PMQ) correlated to job satisfaction, workload, access to educational resources and quality of supervision (8), therefore contributing to the variation in career progression. There was no published data on the impact of disability in progression, although there was anecdotal data to suggest that this was indeed a significant determinant. (9)(10)

\section{Gender}

More women opted for training using the flexible or LTFT pathway, which inevitably extended their training (11-13) and led to significant pay differential (19\% lower) compared to their male peers (14). Surveys of doctors 10 years after graduation found that $42 \%$ of women compared to only $7 \%$ of men, had worked in a LTFT capacity. (15) The highest rates were observed among doctors training in primary care $(\sim 10$ and 6 times greater than non-GPs, respectively), and among those not in training or senior positions.

\section{Race \& Ethnicity}

Race and ethnicity were important determinants of career progression, where those from Black and minority ethnic (BAME) groups faced bigger challenges, reduced opportunities, lower success rates for their applications and lesser likelihood of to reaching their career goals. Data from the Workforce Race Equality Standard (WRES) reports, consistently suggested that fewer than half of BAME doctors perceived that their organisation provided equal opportunities for their career progression (16). A report from the Royal College of Physicians (17) concluded that $98 \%$ of white applicants were likely to be shortlisted at their first application, and $29 \%$ offered a post, compared with $91 \%$ for BAME applicants being shortlisted and only $12 \%$ received similar offers.

Training pathways

\section{LTFT trainees}

Doctors who chose to train flexibly due to a variety of reasons including parenthood, caring responsibilities, personal or family health challenges faced deterrents due to the scarcity of LTFT posts/ opportunities in many specialties. They faced an increased risk of 'burnout' (18), stress (19), which prompted many doctors to take career breaks (e.g. after Foundation Year 2 (FY2) or even forcing some to leave the profession with devastating personal and financial consequences (18) as well as a negative impact on the sustainability of the healthcare workforce.

Furthermore, the small number of who were successful in securing flexible training positions often reported prejudice, being undermined or that their professional opinion was not valued by the rest of the team $(15,18,20,21)$. Private time for study, examination preparation or opportunity to attend professional meetings/ conferences can be reduced or completely foregone by LTFT doctors (21). Interestingly, the Royal College of Physicians Report (17) showed that in medical specialties, working LTFT did not adversely affect the chance of being appointed to a substantive consultant post.

Currently, funding for LTFT posts is severely limited in certain areas such as surgical training or in Cardiology (15), thus creating unbalanced workforce gender distributions and thus negative stereotypes. Even when it is available, LTFT training may not be adequate in supporting trainees to achieve required competences and to gain adequate experience. There is also evidence to suggest that trainees choose not to pursue LTFT training due to fear of discrimination and that the scarcity of sufficient flexible training opportunities disproportionately affect female doctors, who may also lack appropriate role models (11).

\section{Non-training grades}

Doctors who are not in formal training posts, do also have similar education and training requirements, in order to progress within their chosen specialties. However, the evidence suggests that this group receives less to no support and extremely limited access to educational opportunity, compared to those in accredited training posts (22). International medical graduates (IMG) and BAME doctors are less likely to be successful in securing training posts, hence are disproportionately represented in non-training grades including SAS/ LEDs, leading to additional disadvantage (23). Until 2020, the Certificate of eligibility of specialist registration (CESR) was a formal route to get accreditation for non-training grade doctors, based on a demonstration of having acquired equivalence of training, where the burden of proof usually rested on the applicant and the process is strife with 
bureaucracy as well somewhat perception of unsurmountable, logistical challenges. However, the success in CESR/ CCT applications varies significantly for specialties often based on competition ratios, fill rates and efficacy of the assessment process (24).

\section{Causes of DA}

The observed DA in postgraduate medical education and careers in the UK is multifactorial in origin. (25) The literature demonstrates 5 principal determinants: acculturation, bias, identity, social capital and (lack of) practical support, although these are all interrelated. These causes may apply to individuals with certain protected characteristics or disadvantaged groups. It is difficult to demonstrate a direct causal link between the causes and impact on career progression. We have therefore had to make certain assumptions and inferences -for example that poorer relationships and difficulty with communication, are likely to impede progress.

\section{Acculturation}

In this context, the term acculturation (26) refers to the assimilation of doctors from abroad or nonwhite ethnicity/culture into the prevalent notion of a majority, 'white British or Anglo-Saxon' culture. (27) It is recognised that there is little consensus on a homogenous 'white British culture' amongst the majority populace.(28) Thus this section refers predominantly to IMGs rather than to those who were born and educated in the UK. However, those doctors who are born and raised in the UK, from migrant parents or grandparents are also likely to face similar challenges, as those who have migrated in their adulthood. There are different facets to acculturation, which we outline below.

\section{Linguistics}

IMGs who wish to work in the UK, have to score a high mark in the international English language assessments, even if they may have received their education in English in their home countries.(29) This rule also applies to IMGs from the European Economic Area. However there is a well-recognised perception that IMGs tend to struggle in their communication with patients, who may speak different locally prevalent dialects of English. (3033). Colloquialisms may be particularly difficult, even to those who are native speakers or fluent in spoken English parlance. Some IMGs perceive that their accent may make patients and colleagues doubt their clinical capability, reduce their chances at recruitment during interviews and impede success in viva or clinical examinations.
This challenge is also reflected in the lack of knowledge of prevalent local customs, mannerisms and expectations from interpersonal interactions, when dealing with patients and colleagues. A review from the USA (34) found that IMGs could take a few months to build up their interpersonal skills in clinical settings. IMGs sometimes lack awareness of locally prevalent ethical, legal and cultural norms, and have gaps in their knowledge of GMC's professional standards (35) which could potentially be a factor in career progression (32). Furthermore this lack of cultural intelligence may impede forming supportive relationships with colleagues and patients (32). Thus lead to misunderstandings during social and professional interactions, being labelled as 'problematic', and can deter doctors from reporting their challenges as well as when seeking help. This can also result in incidents of discrimination and racism (36).

\section{Power distance norms}

Like in the UK, the medical profession across the world, attracts people who are hardworking, motivated, intelligent, fiercely ambitious, privileged and have access to much higher socio-economic resources to enable them to succeed in a highly competitive education system. Hence, those who become doctors (except in situations of reservation/ positive discrimination/ widening participation) commonly hail from the relatively affluent strata of society. Therefore in many countries there is often a significant socio-economic gap between doctors and majority of their patients. The universal access to healthcare as provided by the UK NHS and the public service nature of the profession, makes such power-distance gaps less visible. Meeuwesen et al (37) found that the larger a nation's power - distance divide, the more fixed the roles of the doctor and patient are - and an attitude described as "doctor knows best" may prevail (38). Hofstede (39) suggests that in high power-distance countries, those in charge are rarely challenged. Morrow (40) found that the difference in power distance in the doctor - patient relationship was often the largest challenge reported by IMGs, in their transition to working in the UK. IMGs from high power distance countries tend to position themselves as the experts, which might cause difficulty in communication and misunderstanding with patients who may find such interactions arrogant. The understanding of the patient's agenda, the need to seek informed consent and explain all clinical recommendations in plain English parlance can present an unfamiliar landscape for IMGs. Such power-distance challenges may also impact interactions between members of the multi-professional teams.

\section{Awareness of cultural norms}


Bias

\section{Systemic Bias}

Systemic bias is an important determinant of DA that exists in career progression. Sir Michael Marmot describes structural racism and the legacy of colonialism as one of the three structural drivers of health inequality, along with macroeconomic forces including the environment. In the UK, qualitative research has found that racism has direct effects on both mental and physical health. A systematic review of 121 studies on racism and health found evidence of negative effects, particularly relating to mental health. Another study of young people in London found that "unlike other measures of adversity, perceived racism was consistently associated with poorer psychological wellbeing across gender, ethnicity, and age." David Williams suggested that from a statistical, empirical point of view, discrimination was one of the reasons there are racial disparities in health existed. The state of affairs in the NHS which reflects society in general, therefore is not much different.(41)

There is evidence of systemic bias in competitive allocation of foundation school places. Ethnicity, secondary school type (private versus state), being from an area of high educational participation and country of medical qualification are strong predictors of allocation to the preferred choice of Foundation school, in the UK. Those from BAME backgrounds are less likely to be allocated their first-choice Foundation school, even after controlling for the effect of the application score and Foundation school competition ratios. (42)

Staff grade and SAS doctors (who constitute one in five of all doctors with a licence to practice, $29 \%$ being women and $69 \%$ qualified outside the EEA) are recognised victims of poor support, career advice, discriminatory job plans, endure inappropriately high work schedules, have inadequate supervision, receive scant educational opportunities, do not have recourse to developing additional skills, time to develop and little prospects of career progression $(22,43)$.

\section{Unconscious Bias}

Unconscious bias which may not be overt racism can still contribute to the forming of 'in-groups' and 'out-groups' leading to the exclusion of IMGs, BAME doctors, doctors who work less than full time, as well as those with disabilities from opportunities and access to appropriate progression (44). Unconscious bias leads to discrimination in many levels including limiting access to mentorship or sponsorship and therefore directly affects progression (45).

\section{Identity}

IMGs face a number of psychosocial issues such as loss of personal identity and belonging, confusion of roles and responsibilities and even loss of financial autonomy after migration (46). Inclusion has positive effects on social interaction and thus on belonging. It is likely to result in improved selfefficacy. Self-efficacy affects not only behaviour, but also the aspirations people have for themselves (47). Stereotype threat is a well-recognised phenomenon whereby people feel themselves to be at risk of conforming to stereotypes about their social group. It is thought to contribute to racial and gender gaps in academic performance (48).

\section{Social Capital: Relationships with seniors and peers}

Woolf (32) reported that poorer relationships with seniors and problems fitting in at work can lead to fewer learning opportunities, lower confidence, burn out, social isolation, lack of support from work in coping with problems outside work and increased chance of mental health problems. Moving jobs frequently means that there is a shorter time to build relationships and establish any opportunities to follow a role model, which impedes progress (49). Establishing good relationships can take longer due to cultural differences, thus particularly disadvantaging IMGs. BAME UK graduates might also be disadvantaged as they are seen to be less likely to "fit the mould". Doctors often prefer peer support and guidance from others within the same cultural group as a result, IMG's often missed out on social events in which others were given the opportunities to share knowledge and seek emotional support (49).

\section{Practical support}

Woolf found that doctors value emotional and practical support from partners and families, especially when having challenges at work. Working long duty hours, experiencing inflexible training pathways, a perceived lack of understanding from supervisors and the lack $f$ appreciation of a work-life balance are deterrents to success. These factors may be especially relevant for IMGs, women and those with caring responsibilities or disabilities. They are often isolated and separated from their families, friends and social support systems (50). Woolf noted that doctors often lack autonomy about where they work and live. Without other social support, it is likely that this is especially stressful for IMGs. IMGs may also experience financial difficulties for example if they are subject to additional expenses such as being charged for clinical attachments, difficulty in obtaining work permits or visas can limit job opportunities. 


\section{Discussions/solutions}

The UK NHS has attracted the very best of talented medical professionals from across the globe, particularly from countries in the Commonwealth. The roots of such affinity to the UK medical education and healthcare system perhaps lies in the colonial past. The heritage institutions of the medical royal colleges with their global facing departments have propagated the value and respect that a UK medical qualification or training affords in the Commonwealth countries. It is also clear that since its inception in 1948, the UK NHS has been a beacon of universal healthcare provision, irrespective of the ability to pay, which is an envy of many countries. Hence, it is not unusual that medical professionals, who are by definition ambitious, motivated and socially mobile will seek to experience this system first-hand.

In addition, the UK NHS relies on a substantial proportion of its workforce to be trained and recruited from elsewhere. The NHS would not be able to provide the quality healthcare that it aspires to and its clientele expect, without the dedication of professional staff from across the globe. Medical migration also affords a huge economic advantage to the host country at the detriment of the countries of origin. Yet, the systems to induct, integrate and train professionals from 'abroad' is biased and severely deficient. The subsequent treatment of such migrant doctors in the NHS is inexplicable and certainly unfair. There is little general recognition of the richness of cultural experience and clinical diversity that migrant doctors (and other healthcare professionals) bring with them.

It is clear from our review that DA in career progression is a manifestation of systemic bias combined with the additional disadvantage faced by those substantial minority who have qualified and migrated to the UK. Such bias is also experienced by those UK born and educated professionals based on their gender, disability, race or ethnicity.

Although recognised for generations and legally recognised as a phenomenon since the 2014 legal challenge (BAPIO vs Royal College of General Practitioners)(51) followed by the GMC declaration in 2015, there is little evidence of understanding the causes, consequences and mitigation.

This review has also highlighted that there is scarcely any published data, monitoring of career progression or responsibility for the plight of thousands of doctors who are in the underrepresented minority at government or regulatory level. Health Education England since its existence has been focussed on the improvement of the learning environment, working with the medical royal colleges has developed and delivered curricula, but until recently has shown little interest or provided any support to the 'non-training grade' doctors. The regulator (GMC) has only started exploring the feedback from non-training doctors, attempting to understand the plight of SAS doctors and recognised that DA in medical professions is an unacceptable manifestation of systemic bias. The governmental agencies have often worked against each other (Home Office regulations vs Department of Health and Social Services) adding to the predicament of healthcare professionals who have answered calls for recruitment 'to keep the NHS from collapsing'. Doctors unions and support organisations such as the British Medical Association have little meaningful contribution to the improvement of standards of education, training and employment and addressing the bias that has existed for the disadvantaged minority.

However, there is change in the air and since the establishment of the WRES benchmarking, the GMC National Training Surveys, HEE National Education and Training Surveys there is a realisation that all is not well. There is a renewed interest in the training, support and wellbeing of all doctors. There is a new interest in the plight of the under-represented and discriminated substantial minority. The gender pay gap, the disadvantages of flexible training and the bias in career progression is no longer destined to be in the darkness of oblivion. Here is an opportunity to improve data collection, plotting career pathways, provide support, benchmark organisations and hold them accountable and above all create the environment for meaningful dialogue with this group of disadvantaged workforce.

Here are some of the initiatives that our review brought to the front and may hold promise.

\section{Training environment:}

The GMC commissioned report 'What supported your success in training' highlights that solutions for DA must have a broad focus, beyond examination bias, on the training environment itself and allow individuals support and training requirements to be identified (52). Championing better work and working environment has been recommended in guidance for employers and policy makers, to improve experience, involvement and opportunities for BAME or disadvantaged employees (53).

We have been able to draw up a list of key priorities in the training environment: 
- To ensure that understanding of differential attainment is improved at a local, regional and national level, in order to drive remedial action.

- To encourage flexibility and responsiveness, whereby training is tailored to the individual requirements of each doctor.

- To involve groups of doctors who have struggled personally with career progression, in designing improvements to training delivery. This may lead to a more equitable training environment and promote trainee self-efficacy.

- To provide organisational support to less advantaged groups of doctors to help them to achieve their goals -for example those who have qualified from abroad, those whose first language isn't English, doctors in non-training grades and those working less-than-full time.

\section{Minimising /mitigating Bias}

Unconscious/Implicit bias training has been implemented in multiple sectors and industries as a means of addressing discrimination. Such training may seek to achieve this by raising awareness, reducing implicit bias, reducing explicit bias and changing behaviour. A review conducted by the Equality and Human Rights Commission into the impact of bias training concluded that, whilst commonly used bias training strategies are likely to increase awareness and reduce implicit bias, there is limited evidence for efficacy in engendering behaviour change. This paucity of evidence was ascribed to a lack of valid measures of behaviour change in the published literature (54). There may also be a risk of counterproductively legitimising bias if the training is perceived to normalise the phenomenon of unconscious bias (55). Devine et al. suggest that the impact of bias training may be especially limited if the recipients are not in a receptive state to challenge their biases.(56) These observations emphasise the need for colleagues conducting future work in this area to carefully and deliberately consider the context, content and evaluation method when implementing unconscious bias training.

\section{Supporting disadvantaged groups}

The 2014 Royal College of Psychiatrist's IMGs conference highlighted the importance of employers providing substantive, tailored induction programs for international doctors new to the UK (57). Kehoe et al. (58) report that ongoing organisational and training support is needed in order to optimally engage IMGs in training and maximise their self-efficacy and cultural capital. They advocate that a support program to achieve professional and cultural adjustment would ideally involve synthesis of multiple elements including individual needs assessments, role modelling, supervised practice, social networking and ongoing supervisor and peer support.

HEE is now offering many of these recommended forms of support to doctors returning to training schemes after taking any form of career break e.g. sickness, parental leave, research, work abroad etc. The Supported Return To Training (SuppoRRT)(59) scheme offers accelerated learning, refresher courses, supernumerary working, mentoring and enhanced supervision. The need for wider access to this support for other groups is recognised and nationally there is a move to make the SuppoRRT scheme and resources available to IMGs entering training and even those in non-training roles such as CaReforMe.

Co-production of training and Inclusive training delivery

Differential attainment, across groups of trainees from different cultures, may relate in part to differences in the values ascribed by the learners and by the providers, to learning experiences. Coproduction of training with the active involvement of less well-performing groups in curriculum development "through the identification of the learning needs and/or preferences of the learner" may be an innovative approach (60). This inclusive approach to training may foster a greater sense of belonging in training and facilitate more equitable access to achievement and progression. Shah and Ahluwalia (47) further develop this notion, suggesting that the sense of belonging that results from inclusive delivery of training which "values and capitalises on different approaches", may enhance trainees' self-efficacy. This improved perception of their own abilities may then translate into the setting of aspirations for progression and the confidence with which to achieve it.

Other groups, beyond doctors from other cultures and ethnic groups, may also stand to benefit from a more inclusive approach to training that considers their individual needs and experiences. A large regional survey highlighted the need for deaneries, and employing trusts, to adopt policies that support SAS doctors' career progression. Suggestions included SAS doctor inclusion in annual appraisal, universal provision of a formal educational supervision and facilitation of use of the study leave allowance (61). 


\section{Tailored approach for LTFT}

Flexible and LTFT training broadens opportunities to achieve Certificate of Completion of Training (19). Recently published consensus recommendations from The Association of Surgeons in Training (ASiT) suggest that expanded availability of LTFT training and increased awareness of flexible training opportunities could improve access and career progression, particularly for women and those needing to prioritise family responsibilities (11). ASiT emphasise the need to tailor LTFT posts to meet the training requirements of the individual. Practical suggestions include:

- Providing mentors with experience of LTFT training

- When allocating jobs, Training Programme Directors should consider the career and health reasons for choosing LTFT training.

- Allowing workplace based assessments to be completed pro-rata according to the percentage of LTFT worked

- Avoiding simultaneously training a higher LTFT trainee alongside another higher trainee during a key training session (e.g. a surgical list)

Jones et al (19) suggest that there is currently a knowledge gap surrounding our understanding of career progression of LTFT trainees who achieve CCT but do not apply for substantive consultant appointments. They suggest that monitoring of members via annual surveys would allow Royal Colleges to better understand post-CCT career progression as undertaken by the RCP in 2020 .

A key barrier to progress in addressing differential attainment, is a paucity of evaluation data for attempted interventions. Acknowledging this, the GMC have established a portal for sharing case studies of effective practice (62). The GMC has worked to raise the political profile of differential attainment by incorporating it into its quality assurance framework. Regional differential attainment data is provided to HEE Deans who must then respond with an action plan and demonstrate how they intend to evaluate their interventions.

Potential solutions and knowledge gap to explore further:

There are still many unanswered questions which require reflection and strategic planning:

- How can cultural awareness become embedded in clinical practice?

- How can stakeholders or trusts best provide practical support to IMGs?
- How can IMGs doctors best access support should they feel discriminated against by their peers?

- How can we establish a system at every workplace to create a diverse group of professionals network, both formally and informally, especially when they are new to their workplace?

- Does every doctor have access to an allocated mentor if support is needed? If so, how can the quality of this relationship be assured? Do allocated mentors have appropriate knowledge and understanding of the particular challenges faced by the groups of doctors whom they support?

- Which additional steps should be considered in curriculum delivery or training to improve trainees' success, experiences and outcomes?

- How can we increase access to flexible training whilst ensuring equitable career development opportunities?

- How can we monitor progress in the postCCT period and who should own responsibility for this?

- How can we collect and share more granular data, providing a broader focus on protected characteristics and the intersectionality of these on career progression?

\section{Summary}

If our system of medical training afforded sufficient support and opportunity for each doctor to individually succeed, the injustice of differential attainment in career progression would cease to exist. This pernicious issue is complex and multilayered, with far reaching consequences for groups not favoured by 'the status quo'. The system itself is also a casualty, deprived of the talent, voices and contributions of the doctors who fall behind. There is a moral and legal duty to set things right. Solutions will not be simple or singular. Success will require collaborative working both within and between organisations. Co-production of solutions with the affected groups should be prioritised. In moving from an 'action planning' to an action phase, interventions must be designed to enable the evaluation of their impact. Differential attainment is a disease that will require evidence based treatment.

\section{Limitations}

This scoping document is a rapid review of literature from Google scholar, PubMed and the various organisation reports. There is a possibility that some actions are already in place at various 
workplaces but have not been followed up or published.

\section{References:}

1. Sheikh Z. Increased working hours, simulation technology or competency based progression? What is the solution for surgical training? Surg Oxf. 2017 Aug 1;35(8):469-72.

2. McArdle J. Medical training pathway [Internet]. The British Medical Association is the trade union and professional body for doctors in the UK. [cited 2020 Dec 29]. Available from: https://www.bma.org.uk/advice-andsupport/studying-medicine/becoming-adoctor/medical-training-pathway

3. Shape of Training and the physician training model [Internet]. JRCPTB. 2015 [cited 2020 Dec 29]. Available from: https://www.jrcptb.org.uk/imt

4. Specialist applications and certificates statistics [Internet]. [cited 2020 Dec 29]. Available from: https://www.gmc-uk.org/about/what-we-doand-why/data-and-research/medical-practicestatistics-and-reports/specialist-applications-andcertificates

5. Out of programme (OOP) [Internet]. [cited 2020 Dec 29]. Available from: https://www.gmcuk.org/education/standards-guidance-andcurricula/guidance/out-of-programme

6. insight-paper---sas-and-leds.pdf [Internet]. [cited 2020 Dec 29]. Available from: https://www.gmc uk.org/-/media/documents/insight-paper---sasand-leds.pdf

7. Protocol for Thematic Synthesis of Differential Attainment in the Medical Profession - 'Bridging the Gap' Series | Sushruta Journal of Health Policy \& Opinion [Internet]. [cited 2020 Oct 31].

Available from:

https://www.sushrutajnl.net/index.php/sushruta /article/view/91

8. The association between trainee demographic factors and self-reported experience: Analysis of General Medical Council National Training Survey 2014 and 2015 data - Dipender Gill, 2016 [Internet]. [cited 2020 Dec 29]. Available from: https://journals.sagepub.com/doi/10.1177/2054 270416632705

9. Doctors with disabilities [Internet]. Health Careers. 2015 [cited 2020 Dec 29]. Available from: https://www.healthcareers.nhs.uk/exploreroles/doctors/career-opportunitiesdoctors/doctors-disabilities

10. Patterson C. Disabled doctors struggle with inclusivity, finds survey [Internet]. The British Medical Association is the trade union and professional body for doctors in the UK. [cited 2020 Dec 29]. Available from:

https://www.bma.org.uk/news-andopinion/disabled-doctors-struggle-withinclusivity-finds-survey

11. Less Than Full-time Training in surgical specialities: Consensus recommendations for flexible training by the Association of Surgeons in Training - ScienceDirect [Internet]. [cited 2020 Dec 29]. Available from: https://www.sciencedirect.com/science/article/p ii/S1743919115012030

12. Skinner H, Bhatti F. Women in surgery. Bull R Coll Surg Engl. 2019 Aug 31;101(suppl_6):12-4.

13. How to reduce the stress of general dental practice: The need for research into the effectiveness of multifaceted interventions | British Dental Journal [Internet]. [cited 2020 Dec 29]. Available from:

https://www.nature.com/articles/4813463

14. Independent review into gender pay gaps in medicine in England [Internet]. GOV.UK. [cited 2020 Dec 29]. Available from:

https://www.gov.uk/government/publications/i ndependent-review-into-gender-pay-gaps-inmedicine-in-england

15. Lachish S, Svirko E, Goldacre MJ, Lambert T. Factors associated with less-than-full-time working in medical practice: results of surveys of five cohorts of UK doctors, 10 years after graduation. Hum Resour Health. 2016 Oct 13;14(1):62.

16. NHS England » Workforce Race Equality Standard data reporting - 2019 [Internet]. [cited 2020 May 24]. Available from:

https://www.england.nhs.uk/publication/workfo rce-race-equality-standard-data-reporting-2019/

17. 2019 survey of medical certificate of completion of training (CCT) holders' career progression [Internet]. RCP London. 2020 [cited 2020 Dec 29]. Available from:

https://www.rcplondon.ac.uk/projects/outputs/ 2019-survey-medical-certificate-completiontraining-cct-holders-career-progression

18. Illsley A. Time to look more closely at attitudes to less than full time working? Br J Hosp Med. 2020 Feb 2;81(2):1-3.

19. 'Mainstreaming' of less than full-time training Jones - 2015 - Anaesthesia - Wiley Online Library [Internet]. [cited 2020 Dec 29]. Available from: https://associationofanaesthetistspublications.onlinelibrary.wiley.com/doi/full/10. 1111/anae.13096

20. Part-time working: full-time professionalism | RCP Journals [Internet]. [cited 2020 Dec 29]. Available from:

https://www.rcpjournals.org/content/clinmedici ne $/ 9 / 6 / 560$

21. Rickard C, Smith T, Scallan S. A comparison of the learning experiences of full-time (FT) trainees and less than full-time (LTFT) trainees in general practice. Educ Prim Care. 2012 Jan 1;23(6):399_ 403.

22. Rippin H, Buckley EG. The educational needs of staff grade doctors and dentists in Scotland. Health Bull (Edinb). 1996 Jul;54(4):318-31.

23. sas-and-le-doctors-survey-initial-findings-report060120_pdf-81152021.pdf [Internet]. [cited 2020 Dec 29]. Available from: https://www.gmcuk.org/-/media/documents/sas-and-le-doctorssurvey-initial-findings-report-060120_pdf81152021.pdf

24. Retrospective granting of CCT to doctors granted a CESR through the combined programme [Internet]. [cited 2020 Dec 29]. Available from: https://www.gmc-uk.org/registration-and- 
licensing/join-the-register/registrationapplications/specialist-applicationguides/retrospective-cct-for-doctors-with-cesrcombined-programme

25. Nunn S. Understanding differential attainment across medical training pathways: A rapid review of the literature. :89.

26. Schwartz SJ. Rethinking the concept of acculturation: Implications for theory and research. [Internet]. Vol. 65, American Psychologist. US: American Psychological Association; 20100510 [cited 2020 Dec 29]. p. 237. Available from: /fulltext/2010-08987001.html

27. Brown R, Zagefka H, Tip LK. Acculturation in theUnited Kingdom. In: Sam DL, Berry JW, editors. The Cambridge Handbook of Acculturation Psychology [Internet]. 2nd ed. Cambridge: Cambridge University Press; 2016 [cited 2020 Dec 29]. p. 375-95. (Cambridge Handbooks in Psychology). Available from: https://www.cambridge.org/core/books/cambri dge-handbook-of-acculturationpsychology/acculturation-in-theunitedkingdom/DB4DA8C6081A32B562D6948FF41701 AA

28. Culture of the United Kingdom. In: Wikipedia [Internet]. 2020 [cited 2020 Dec 29]. Available from:

https://en.wikipedia.org/w/index.php?title=Cultu re_of_the_United_Kingdom\&oldid=995438707

29. Mallows D, British Council. Language issues in migration and integration: perspectives from teachers and learners. 2014

30. Woolf K. Fair Training Pathways for All: Understanding Experiences of Progression - Final Report. :69.

31. Rich A, Viney R, Needleman S, Griffin A, Woolf K. 'You can't be a person and a doctor': the work-life balance of doctors in training-a qualitative study. BMJ Open [Internet]. 2016 Dec 1 [cited 2020 Jan 21];6(12). Available from: https://bmjopen.bmj.com/content/6/12/e01389 7

32. Woolf K, Viney R, Rich A, Jayaweera H, Griffin A. Organisational perspectives on addressing differential attainment in postgraduate medical education: a qualitative study in the UK. BMJ Open [Internet]. 2018 Mar 9 [cited 2020 Jan 21];8(3). Available from:

https://www.ncbi.nlm.nih.gov/pmc/articles/PMC 5855204/

33. Humphries N, Tyrrell E, McAleese S, Bidwell P, Thomas S, Normand C, et al. A cycle of brain gain, waste and drain - a qualitative study of non-EU migrant doctors in Ireland. Hum Resour Health. 2013 Dec 9;11(1):63.

34. Singhal K, Ramakrishnan K. Training Needs Of International Medical Graduates Seeking Residency Training: Evaluation Of Medical Training In India And The United States. Internet J Fam Pract [Internet]. 2003 Dec 31 [cited 2020 Dec 29];3(1). Available from:

https://ispub.com/IJFP/3/1/8127

35. Khan FA, Chikkatagaiah S, Shafiullah M, Nasiri M, Saraf A, Sehgal T, et al. International Medical
Graduates (IMGs) in the UK-a Systematic Review of Their Acculturation and Adaptation. J Int Migr Integr. 2015 Aug 1;16(3):743-59.

36. Jones L, Halford S, Leonard P. Racism in the medical profession: the experience of UK graduates. 2003.

37. Meeuwesen L, van den Brink-Muinen A, Hofstede G. Can dimensions of national culture predict cross-national differences in medical communication? Patient Educ Couns. 2009 Apr;75(1):58-66

38. Verma A, Griffin A, Dacre J, Elder A. Exploring cultural and linguistic influences on clinical communication skills: a qualitative study of International Medical Graduates. BMC Med Educ. 2016 Jun 10;16(1):162.

39. Cooper CL. Culture's Consequences: International differences in work related values, Geert Hofstede Sage Publications, London and Beverly Hils, 1980. No. of pages: 475. Price £18.75. J Organ Behav. 1982;3(2):202-4.

40. Morrow G, Rothwell C, Burford B, Illing J. Cultural dimensions in the transition of overseas medical graduates to the UK workplace. Med Teach. 2013 Oct 1;35(10):e1537-45.

41. Anekwe L. Harnessing the outrage: it's time the NHS tackled racial bias. BMJ. $2020 \mathrm{Feb}$ 11;368:m341.

42. Relationship between sociodemographic factors and selection into UK postgraduate medical training programmes: a national cohort study BMJ Open [Internet]. [cited 2020 Dec 29]. Available from:

https://bmjopen.bmj.com/content/8/6/e021329

43. Oroz C, Sands LR, Lee J. SAS doctors career progression survey 2013. Int J STD AIDS. 2016 Mar 1;27(3):231-4.

44. Lieberman MD, Rock D, Halvorson HG, Cox C. BREAKING BIAS UPDATED: THE SEEDS MODEL ${ }^{\mathrm{TM}}$ 2015;19.

45. Ng TWH, Eby LT, Sorensen KL, Feldman DC. Predictors of Objective and Subjective Career Success: A Meta-Analysis. Pers Psychol. 2005;58(2):367-408.

46. Wong A, Lohfeld L. Recertifying as a doctor in Canada: international medical graduates and the journey from entry to adaptation. Med Educ. 2008 Jan;42(1):53-60.

47. Shah R, Ahluwalia S. The challenges of understanding differential attainment in postgraduate medical education. Br J Gen Pract J R Coll Gen Pract. 2019 Sep;69(686):426-7.

48. Mountford-Zimdars A, Sanders J, Moore J, Sabri D, Jones S, Higham L. What can universities do to support all their students to progress successfully throughout their time at university? Perspect Policy Pract High Educ. 2017 Jul 3;21(2-3):10110.

49. Woolf K, Rich A, Viney R, Needleman S, Griffin A. Perceived causes of differential attainment in UK postgraduate medical training: a national qualitative study. BMJ Open. 2016 25;6(11):e013429.

50. Woolf K, Rich A, Viney R, Needleman S, Griffin A. Perceived causes of differential attainment in UK postgraduate medical training: a national 
qualitative study. BMJ Open [Internet]. $2016 \mathrm{Nov}$ 25 [cited 2020 Jul 8];6(11). Available from: https://www.ncbi.nlm.nih.gov/pmc/articles/PMC 5168507/

51. R (BAPIO) v Royal College of General Practitioners \& General Medical Council [2014] EWHC 1416 (Admin) [Internet]. Equally Ours. [cited 2020 Aug 18]. Available from:

https://www.equallyours.org.uk/0033-r-bapio-vroyal-college-of-general-practitioners-generalmedical-council-2014-ewhc-1416-admin/

52. final-report-success-factors-in-training-211119pdf_pdf-81634780.pdf [Internet]. [cited 2020 Nov 10]. Available from: https://www.gmc-uk.org//media/documents/final-report-success-factorsin-training-211119-pdf_pdf-81634780.pdf

53. addressing-the-barriers-to-BAME-employeecareer-progression-to-the-top_tcm18-33336.pdf [Internet]. [cited 2020 Dec 29]. Available from: https://www.cipd.co.uk/Images/addressing-thebarriers-to-BAME-employee-career-progressionto-the-top_tcm18-33336.pdf

54. Atewologun D, Tresh F. Unconscious bias training : An assessment of the evidence for effectiveness [Internet]. 2018 [cited 2020 Jul 8]. Available from: /paper/Unconscious-bias-training-\%3A-Anassessment-of-the-AtewologunTresh/509a580783bb5df10f3af42e8df720d8ad5 $7 \mathrm{dbd} 0$

55. Duguid MM. Condoning stereotyping? How awareness of stereotyping prevalence impacts expression of stereotypes. [Internet]. Vol. 100, Journal of Applied Psychology. US: American Psychological Association; 20141013 [cited 2020
Dec 29]. p. 343. Available from: /fulltext/201443472-001.html

56. Devine PG, Forscher PS, Austin AJ, Cox WTL. Longterm reduction in implicit race bias: A prejudice habit-breaking intervention. J Exp Soc Psychol. 2012 Nov 1;48(6):1267-78

57. Rai Y, Al-Uzri M. Medical Training Initiative (MTI) Psychiatry Scheme: Online survey of trainees' experience. 2020;13(1):5.

58. Kehoe A, McLachlan J, Metcalf J, Forrest S, Carter $\mathrm{M}$, Illing J. Supporting international medical graduates' transition to their host-country: realist synthesis. Med Educ. 2016 Oct;50(10):1015-32.

59. HEE supporting returners to work in the fight against the COVID-19 pandemic [Internet]. Health Education England. 2020 [cited 2020 Mar 21]. Available from: https://www.hee.nhs.uk/newsblogs-events/news/hee-supporting-returnerswork-fight-against-covid-19-pandemic

60. Sandars J, Walsh K. The value of health professions education: the importance of understanding the learner perspective. Educ Prim Care. 2016 Jul 3;27(4):254-7.

61. Brown J, Kirton J, Shaw NJ, Murphy P, Clarke R. The training needs and career intentions of staff grade and associate specialist doctors. Br J Hosp Med. 2012 Sep 1;73(9):521-5.

62. Linton S. Taking the difference out of attainment. BMJ [Internet]. $2020 \mathrm{Feb} 12$ [cited $2020 \mathrm{Aug}$ 6];368. Available from: https://www.bmj.com/content/368/bmj.m438 